\section{Bisphosphocins: novel antimicrobials for enhanced killing of drug-resistant and biofilm-forming bacteria}

\author{
Jonathan P Wong ${ }^{*, 1}$, Paul DiTullio² \& Steve Parkinson²
}

\begin{abstract}
The global prevalence of antibiotic resistance and the threat posed by drug-resistant superbugs are a leading challenge confronting modern medicine in the 21st century. However, the progress on the development of novel antibiotics to combat this problem is severely lagging. A more concerted effort to develop novel therapeutic agents with robust activity and unique mechanisms of action will be needed to overcome the problem of drug resistance. Furthermore, biofilm forming bacteria are known to be increasingly resistant to the actions of antibiotics and are a leading cause of mortality or morbidity in nosocomial infections. Bisphosphocins (also scientifically known as nubiotics) are novel small protonated deoxynucleotide molecules, and exert their antibacterial activity by depolarization of the bacterial cell membrane, causing bacterial cell death. Bisphosphocins may represent an effective weapon against antibiotic-resistant and biofilm-forming pathogenic bacteria. Preclinical efficacy studies in animals have shown that the compounds are safe and, efficacious against various bacterial infections, including drug-resistant pathogens. In vitro biochemical analysis confirmed that the bactericidal activity of bisphosphocins is mediated by depolarization of the bacterial cell membrane, and these compounds are better able to penetrate through bacterial biofilm and kill the biofilm encased bacteria. This article will cover the structure, mode of action, safety, efficacy and the current state of development of bisphosphocins. Together, the information presented here will present a strong case for bisphosphocins to be considered for use as new weapons to complement the existing arsenal of antimicrobial drugs and as a first line defence against drug-resistant and biofilmforming bacteria.
\end{abstract}

The WHO identified antimicrobial resistance as one of the three greatest threats to human health [1]. The most serious concern regarding antibiotic resistance is that some clinically important bacteria have become resistant to most, if not all, of the commonly available antibiotics. These bacteria pose a serious threat to public health and are a leading cause of mortality. Bacteria which fall into this category are considered to be 'superbugs' and they include methicillin-resistant Staphylococcus aureus (MRSA), vancomycin-resistant Enterococcus (VRE) and multidrug-resistant Mycobacterium tuberculosis (MDR-TB). While the prevalence of antibiotic resistance has rapidly increased globally, $\mathrm{R} \& \mathrm{D}$ funding for the development of new antibiotics by pharmaceutical companies, ironically, has markedly decreased. This has resulted in a global crisis in that severely ill infected patients are left with fewer and fewer treatment options resulting in poor clinical outcomes [2]. To address this crisis, infectious disease experts and public health leaders are developing numerous creative incentives in an attempt to stimulate new antibacterial R\&D. For example, the $10 \times 20$ initiative

'Defence Research \& Development Canada, Suffield Research Center, Ralston, Alberta, Canada

2Lakewood-Amedex Inc., 3030 University Pkwy, Sarasota, FL 34243, USA

*Author for correspondence: Tel.: +1 403544 4689; Fax: +1 403544 3388; jonathan.wong@drdc-rddc.gc.ca

\section{KEYWORDS}

- antibiotic resistance

- biofilm • bisphosphocins

- broad spectrum

- membrane depolarization

- treatment 
was championed by Infectious Disease Society of America and other infectious disease organizations with the ambitious goal to develop ten new, safe and effective antibiotics by 2020 [3]. The success of such a noble initiative will require concerted efforts by scientists across a multitude of disciplines, be difficult to achieve and require novel and innovative approaches.

Compounding this antibiotic resistance problem in infectious disease chemotherapy is the growing evidence that biofilm forming bacteria are associated with the majority of chronic infections [4]. These clinically difficult to treat infections involve bacteria that produce biofilm which tightly binds the bacteria, rendering them resistant to the inhibitory action of antibiotics because the bacteria become sessile or dormant. In addition, the biofilm makes the bacteria resistant to phagocytosis and other actions of other immune defense mechanisms normally employed by the body to eradicate an infection. It is now estimated that about $65 \%$ of nosocomial infections are associated with biofilm-causing bacteria [5], creating a significant burden on the healthcare system.

Bisphosphocins are experimental antibacterial agents which have been shown in vitro to be bactericidal against a wide range of Grampositive, Gram-negative and acid-fast bacteria [6]. These molecules have been shown to be safe and well tolerated in experimental animal studies, and highly efficacious in the treatment of bacterial infections in various animal infection models, including skin wound and pulmonary Pseudomonas aeruginosa infections. The results from animal studies affirm the potential role of these molecules to treat acute and chronic infections caused by drug-resistant and biofilm-forming bacteria. Here we will cover the structures, mechanism of action of bisphosphocins and highlight the antibacterial efficacy in animal studies against $P$. aeruginosa infection, a clinically important pathogen which can readily develop drug resistant to conventional antibiotics, and produce biofilm to resist the action of antibiotics.

\section{Structure}

Bisphosphocins are synthetic small molecule deoxynucleotides and derivatives whose chemical structures are shown in Figure 1. These molecules are fully protonated for their enhanced uptake by bacterial cells. Bisphosphocins are a novel class of synthetic broad-spectrum antimicrobials that have been chemically designed to have longterm stability, $\mathrm{pH}$ stability and be resistant to nuclease degradation. The lead compound in this classis $\mathrm{Nu}-3$, thymidine 3',5'-bis-(n-butylphosphate) $-5^{\prime}$ and is being developed to treat a number of serious acute and chronic bacterial infections.

\section{Mechanism of antibacterial activity}

Unlike conventional antibiotics with antibacterial activity mediated through inhibition of protein or nucleic acid synthesis, enzymatic degradation and bacterial efflux of drugs, bisphosphocins' mechanism of action is unique and their antibacterial activity is broad spectrum. Experimental studies by Cao et al. [7] using Staphylococcus aureus have shown that bacterial cells treated with $\mathrm{Nu}-3$ showed rapid depolarization of the bacterial cell membrane, as measured using a BacLight ${ }^{\mathrm{TM}}$ Bacterial Membrane Potential Kit from Invitrogen. The significant reduction in the magnitude of this bacterial cell membrane was associated with bacterial cell disruption, which was confirmed by Flow Cell Sorting in the Nu-3 treated bacterial cells. While this study affirmed that bisphosphocins such as NU-3 caused bacterial cell death mediated by cell membrane depolarization, it is not known whether there is other yet to be determined mechanisms that play a role in the antibacterial activity of bisphosphocins. In vitro studies have confirmed that bisphosphocins are directly bactericidal with exposure times of less than $5 \mathrm{~min}$ resulting in 100\% killing [8].

\section{In vitro antibacterial activity against biofilm forming bacteria}

Preliminary studies were also carried out by Akiyoshi et al. [8] to evaluate the effectiveness of bisphosphocins to biofilm forming strains of three bacteria: Acinetobacter baumannii, Klebsiella pneumoniae and Staphylococcus epidermidis using a time kill assay to assess the postantibiotic effect (PAE). Nu-3 exhibited a rapid bactericidal effect on biofilm-encased bacteria with a $100 \%$ kill of all four bacterial strains observed at $170 \mathrm{U} / \mathrm{ml}$ and with a short exposure time of only $10 \mathrm{~min}$. This result further supports experimental data showing $\mathrm{Nu}-3$ to be directly bactericidal through a mechanism of action involving depolarization of the cell membrane which is in contrast to most traditional antibiotics. Furthermore, $\mathrm{Nu}-3$ displayed a rapid $100 \%$ kill rate for both $S$. epidermidis and $A$. 


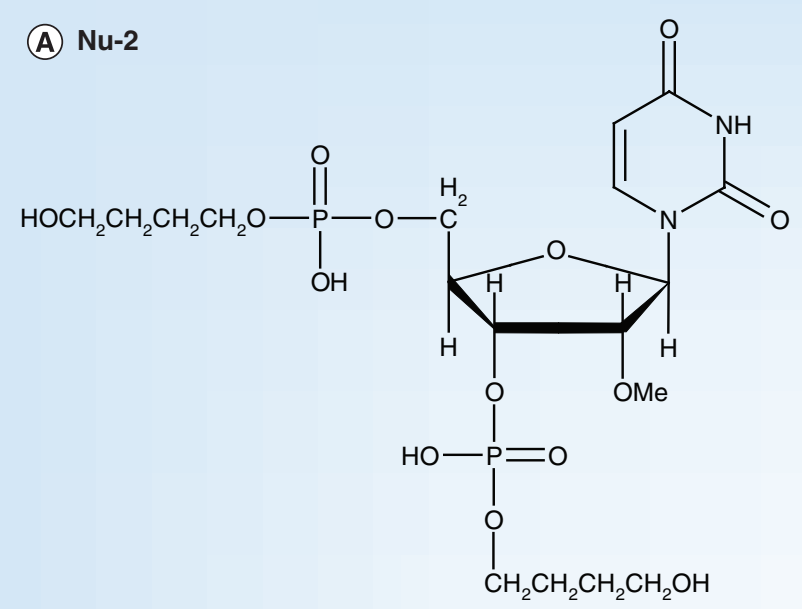

(B) Nu-3

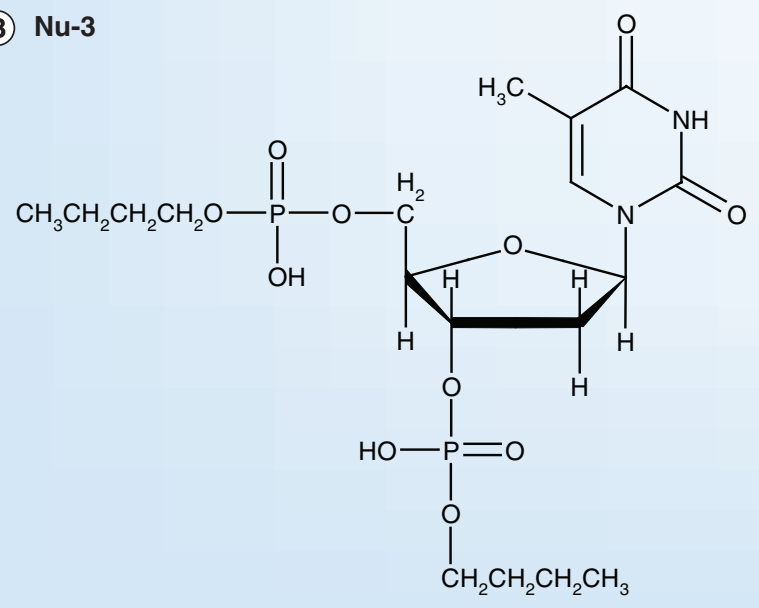

(C) $\mathrm{Nu}-4$<smiles>CCCOP(=O)(O)OC[C@H]1O[C@@H]2C[C@H]1[C@H](OP(=O)(O)OCCC)[C@H]2C</smiles>

(D) Nu-5<smiles>CCCCOP(=O)(O)OCCOCCOP(=O)(O)OCCC</smiles>

\section{Figure 1. Chemical structure.}

baumannii at $170 \mathrm{U} / \mathrm{ml}$ following exposure time of only $5 \mathrm{~min}$ [8]. The rapid kill rate has been shown to be dose and exposure time dependent in contrast to conventional antibiotics. These findings suggest a unique mechanism of rapid killing of biofilm-encased bacteria.

\section{Preclinical studies of efficacy evaluation against antibiotic-resistant \& biofilm- forming bacteria in animals}

The efficacy of bisphosphocins was evaluated against a number of antibiotic-resistant and/ or biofilm forming bacteria using established animal infection models. These studies are important to determine whether the rapid in vitro bactericidal action through cell membrane depolarization/disruption exhibited by bisphosphocins can be exploited as potential broadspectrum therapeutics against clinically relevant pathogens, including $P$. aeruginosa.
Pseudomonas aeruginosa is a Gram-negative, coccobacillus bacterium that is part of the normal skin flora, and is common in the natural environment. It is an opportunistic pathogen and infects respiratory and urinary tracts, burns and wounds of immunocompromised human hosts. For example, patients with burns and wounds are susceptible to $P$. aeruginosa infections in the skin and wounds, and cystic fibrosis patients are predisposed to $P$. aeruginosa infection of the lungs. The therapeutic effectiveness of antibiotic chemotherapy in these patients has been undermined by widespread emergence of antibiotic resistance of $P$. aeruginosa, as well as the ability of $P$. aeruginosa to produce biofilm which forms an effective barrier to antibiotic penetration and activity. Bisphosphocins have been extensively evaluated against $P$. aeruginosa infections using established animal models of $P$. aeruginosa-induced burn wound, and pulmonary cystic fibrosis infections. 


\section{- Efficacy evaluation against burn wound infections}

Infection of the skin and skin structures occur frequently in patients with surgical wounds, burns and other exposed tissues. Pseudomonas aeruginosa is a major opportunistic bacterium that can colonize and infect burn and wound sites, and from there, can rapidly disseminate into distant organs via the bloodstream. In the process, the bacteria can produce a number of virulence factors that can result in sepsis and endotoxic shock. In case studies involving burn patients with $P$. aeruginosa septicemia, the mortality rate was greater than $75 \%$ [9].

A burn wound infection model using mice for the efficacy testing of bisphosphocins was conducted employing a highly pathogenic burn wound clinical isolate of $P$. aeruginosa [10]. Lethal doses of the bacteria were determined for two routes of infection (subcutaneous and topical), representing systemic and local forms of burn wound infections in these burn-induced animals, respectively. Various groups of $P$. aeruginosa infected animals were treated with bisphosphocins administered systemically by intravenous or subcutaneous injection or given topically to the affected sites in the skin.

Bisphosphocins were found to be efficacious for the treatment of topical and systemic forms of burn wound infections and provided 90-100\% survival when treated with NU-2, $\mathrm{Nu}-3$ and $\mathrm{Nu}-4$, compared with $0 \%$ survival in the saline control group [10]. Their therapeutic effectiveness was also affirmed by the sterile bacteriological loads in the livers, spleens and blood samples harvested at day 7 postinfection. By contrast, saline control treated mice were found to exhibit $>2 \times 10 \log _{10} 6$ colony forming units of $P$. aeruginosa [10].

In a related study, the therapeutic efficacy of Nu-3 against $P$. aeruginosa and $S$. aureus in a skin suture wound infection in mice was reported [7]. The mouse suture wound infection model was established by implanting contaminated sutures under the skin, representing a common occurrence following accidental or surgical wounds and trauma. In this study, mice with suture wounds were infected with the bacteria, and then treated with various concentrations of $\mathrm{Nu}-3$ dissolved in $60 \%$ glyecerin solution, with $60 \%$ glycerine in PBS, or with ciprofloxacin ointment. At day 5 post-treatment, the mice were euthanized, and the skin wounds were excised, homogenized and cultured for growth for both bacteria [7].
Results from this study showed the therapeutic effect of Nu-3 in inhibiting the growth of both $P$. aeruginosa and $S$. aureus in the suture wounds in a dose-response manner [7]. Treatment with 1 and $5 \% \mathrm{Nu}-3$ resulted in $1 \log _{10}$ and $2 \log _{10}$ CFU bacterial reductions, respectively, when compared with the PBS control group ( $p<0.01$ and $\mathrm{p}<0.001$, respectively). Treatment with $\mathrm{Nu}-3$ showed similar level of therapeutic effectiveness in this model as the ciprofloxacin ointment [7].

Taken together, these findings affirm the therapeutic efficacy of bisphosphocins against infections of burn and surgical wound infections by two different clinically relevant strains of bacteria.

\section{- Efficacy evaluation against pulmonary cystic fibrosis model}

Respiratory tract infections caused by $P$. aeruginosa are one of the major causes of mortality and morbidity in cystic fibrosis (CF) patients. Prolonged antibiotic treatment by oral or intravenous routes is required to control the severity and duration of pulmonary exacerbation, but despite prolonged and aggressive antibiotic treatment, $P$. aeruginosa infections are rarely completely eradicated in these patients [11]. It is thought that persistent or dormant bacteria, that are resistant to the effect of antibiotics, remain in the mucus of the lungs and re-emerge as an active infection month after antibiotic therapy is completed. The challenges confronting antibiotic treatments of CF patients are increasing antibiotic resistance of $P$. aeruginosa associated with prolonged use, and the ability of $P$. aeruginosa to grow and develop antibiotic-resistant biofilms in the lungs. These challenges are two primary contributing factors for the lack of therapeutic efficacy of antibiotics in these patients. Bisphosphocins may be ideally suited for the treatment of $P$. aeruginosa infections in CF due to their directly bactericidal mechanism of action. These molecules have a robust and unique mechanism of action that does not readily give rise to drug resistance, have an enhanced ability to penetrate through biofilm and are highly effective in killing stationary or dormant bacteria.

In a proof of concept preclinical study to evaluate the therapeutic effectiveness of bisphosphocins, a rat cystic fibrosis model of chronic $P$. aeruginosa infection was established using bacteria-enmeshed in agar beads [12]. To optimize a sustained level of drug in the lungs and enhanced biofilm penetration, $\mathrm{Nu}-3$ was encapsulated in liposomes and delivered to the lungs through 
nebulization [13]. Liposome delivery of antibiotics have been well documented to cause slow, sustained release of drug into the pulmonary sites of infection and increase drug penetration into $P$. aeruginosa biofilm [14].

In this study, groups of Sprague-Dawley rats were infected intratracheally with a clinical CF isolate of $P$. aeruginosa enmeshed in agar beads $\left(2-10 \times 10^{6}\right.$ enmeshed bacteria per animal). The $P$. aeruginosa strain used in the first part of the study was CF strain $\mathrm{PAO}$ with $\mathrm{MIC}_{90}$ of $0.25 \mu \mathrm{g} / \mathrm{ml}$ for ciprofloxacin (a ciprofloxacin susceptible strain). To ascertain the pathogenicity of bacteria in these rats and to validate the chronic nature of the infection in the lungs, lung samples were aseptically harvested from the various groups of rats at weeks 1-3 postinfection, and bacterial loads were determined by standard culture plating on trypticase soy agar for the enumeration of $P$. aeruginosa. Table 1 shows high bacterial loads in the right lungs which were maintained throughout the 3 weeks of the study, indicating the bacteria were pathogenic and established as a chronic infection in the lungs of these animals.

Using this rat model, the therapeutic efficacy of bisphophocin $\mathrm{Nu}-3$ was evaluated. At 2 weeks postinfection, the rats were treated with a single exposure of liposome-encapsulated (LE) Nu-3 or with phosphate buffered saline, administered to the animals via aerosol inhalation using nebulization (50-min aerosol exposure in a nose-only aerosol chamber, nebulized volume was $16 \mathrm{ml}$ of $1300 \mathrm{~A} / \mathrm{ml}$ of $\mathrm{Nu}-3)$. At 4 days post drug treatment, the rats were euthanized and the lungs were aseptically harvested, homogenized and bacterial counts of the various lung homogenates were plated on trypticase soy agar for the enumeration of $P$. aeruginosa (Table 2).

Results presented from Table 2 indicate that lungs from all five infected rats treated with aerosolized PBS contained high $P$. aeruginosa
CFUs, ranging from $1.2 \times 10^{6}$ to $4.0 \times 10^{8}$ in the right lungs. In contrast, the lungs from rats treated with aerosolized LE Nu-3 showed either significantly reduced CFUs (two of the six rats) or undetectable bacterial growth in the right lungs in four of the six rats.

The second part of this study involved evaluating the efficacy of $\mathrm{LE} \mathrm{Nu-3}$ for the treatment of pulmonary infection in Balb/c mice using a ciprofloxacin-resistant $\mathrm{CF}$ clinical isolate of $P$. aeruginosa (strain $\mathrm{PAO}^{\circledR} \mathrm{P} 4438$ with $\mathrm{MIC}_{90}$ of $9 \mu \mathrm{g} / \mathrm{ml}$ for ciprofloxacin). As with the rat study, the animals were intratracheally infected with the agar bead enmeshed bacteria, and at 2 weeks postinfection, were treated with aerosol inhaled LE NU-3 which was delivered to the animals by nebulization. At 4 days post drug treatment, the animals were euthanized and the bacterial loads of the lungs determined (Table 3). While lungs of all eight mice treated with aerosolized PBS showed moderate levels of bacterial loads of $P$. aeruginosa, nine of ten mice that were treated with aerosolized LE Nu-3 showed an undetectable number of bacteria. There was only one nonresponder mouse (animal number 4 ). These results clearly indicate the treatment with aerosolized LE Nu-3 was highly effective against a chronic pulmonary infection of a highly ciprofloxacin-resistant strain of $P$. aeruginosa. There was no significant decrease in the therapeutic effectiveness of LE Nu-3 when used for treatment of ciprofloxacin-resistant compared with ciprofloxacin-susceptible strain of $P$. aeruginosa.

The results from these studies affirm therapeutic efficacy of aerosolized liposome-encapsulated bisphosphocin $\mathrm{Nu}-3$ against chronic $P$. aeruginosa infection in these animal CF models.

\section{Conclusion}

The global battle against antibiotic-resistant bacteria in clinical medicine requires a comprehensive, multiprong strategy that involves

Table 1. Progression of respiratory $P$. aeruginosa infection in rats.

\begin{tabular}{|llll|}
\hline Group & Animal number & CFU/right lung & CFU/left lung \\
7 days postinfection & 1 & $3.0 \times 10^{6}$ & NG \\
& 2 & $1.2 \times 10^{7}$ & $1.3 \times 10^{3}$ \\
\hline \multirow{2}{*}{ 14 days postinfection } & 1 & $1.6 \times 10^{6}$ & $6.0 \times 10^{2}$ \\
& 2 & $6.8 \times 10^{6}$ & $1.8 \times 10^{3}$ \\
\hline \multirow{2}{*}{1 days postinfection } & 1 & $1.0 \times 10^{6}$ & $\mathrm{NG}$ \\
& 2 & $7.8 \times 10^{6}$ & $2.4 \times 10^{3}$ \\
\hline CFU: Colony forming units; NG: No growth. & \\
Reproduced with permission from [13]. ( Future Medicine 2007.
\end{tabular}




\begin{tabular}{|c|c|c|c|}
\hline Group & Animal number & CFU/right lung & CFU/left lung \\
\hline \multirow[t]{5}{*}{ Aerosolized PBS } & 1 & $8.6 \times 10^{6}$ & NG \\
\hline & 2 & $5.6 \times 10^{7}$ & $3.2 \times 10^{7}$ \\
\hline & 3 & $6.0 \times 10^{5}$ & NG \\
\hline & 4 & $4.0 \times 10^{8}$ & NG \\
\hline & 5 & $1.2 \times 10^{6}$ & $4.0 \times 10^{6}$ \\
\hline \multirow[t]{6}{*}{ Aerosolized LE Nu-3 } & 1 & $4.0 \times 10^{6}$ & NG \\
\hline & 2 & NG & NG \\
\hline & 3 & NG & NG \\
\hline & 4 & NG & NG \\
\hline & 5 & NG & NG \\
\hline & 6 & $6.0 \times 10^{5}$ & $2.4 \times 10^{4}$ \\
\hline
\end{tabular}

cooperation involving public health policy makers, infectious disease experts, practising physicians and pharmaceutical leaders to combat over prescriptions of antibiotics, improve guidelines for antibiotic use, improve sanitation standards and minimize the spread of nosocomial infections. At the same time, new weapons to combat antibiotic-resistant bacteria will need to be developed that should ideally have a robust mechanism of action that does not readily give rise to antibiotic resistance, and have an enhanced ability to penetrate biofilms to kill encased sessile bacteria.

Bisphosphocins are novel class of synthetic broad-spectrum antibacterial agents that, when fully developed clinically, can play an important role in complimenting existing antibiotics in combating drug-resistant bacteria in clinical medicine. Bisphophocins are chemically stable, well tolerated in animals and display rapid bactericidal killing mediated by cell membrane depolarization. Numerous preclinical studies have confirmed the therapeutic efficacy of these drugs for the treatment of difficult to treat infections. Bisphosphocins are efficacious for the treatment of antibiotic-resistant and biofilm-forming bacteria such as $P$. aeruginosa. These molecules are effective in the treatment of acute and skin surface infections such as burn wound infections, as well as for chronic and deep

\begin{tabular}{|lll|}
\hline Table 3. Aerosol treatment of ciprofloxacin resistant $P$. aeruginosa infection in Balb/c mice. \\
\hline Group & Animal number & CFU/lung \\
Aerosolized PBS & 1 & $5 \times 10^{5}$ \\
& 2 & $6 \times 10^{5}$ \\
& 3 & $3 \times 10^{5}$ \\
& 4 & $1 \times 10^{5}$ \\
& 5 & $4 \times 10^{4}$ \\
& 6 & $2 \times 10^{5}$ \\
& 7 & $2 \times 10^{5}$ \\
& 8 & $4 \times 10^{4}$ \\
Aerosolized LE Nu-3 & NG \\
& 1 & NG \\
2 & NG \\
& 3 & $4 \times 10^{4}$ \\
4 & NG \\
& NG \\
& 6 & NG \\
7 & NG \\
8 & NG \\
9 & NG \\
\hline Adapted with permission from [13]. Future Medicine 2007. & \\
\hline &
\end{tabular}


tissue infections such as pulmonary infections in cystic fibrosis. Other antibiotics which share similar mechanism of action of bacterial cell membrane depolarization include daptomycin, a lipopeptide antibiotic and telavancin, a lipoglycopeptide antibiotic, and both of these antibiotics have shown antibacterial activities against drug-resistant pathogens and low incidence of drug resistance $[15,16]$.

A Phase I/IIa clinical trial is planned to assess the safety and tolerability of topically applied $\mathrm{Nu}-3$ in patients with chronic infected wounds in mid-2014. Following a successful Phase I/IIa study, a more comprehensive set of Phase IIb studies are planned to evaluate the therapeutic efficacy of bisphosphocin $\mathrm{Nu}-3$ in a number of acute and chronic topical infections such as burn wound, diabetic foot ulcers and surgical wound infections that can be readily treated locally with topically applied drug. In addition, a Phase I safety dose escalating study is planned for late 2014 to assess the safety and tolerability of intravenously administered $\mathrm{Nu}-3$ in normal healthy human adults. Completion of the Phase I study of IV administered Nu-3 would enable the initiation of a Phase II trial targeting a number of serious bacterial infection indications including $P$. aeruginosa lung infections in cystic fibrosis patients, that could also include delivered directly to the respiratory tract via aerosol inhalation by nebulization.

Bisphosphocins when fully developed clinically will be a potent and broad-spectrum class of novel antimicrobials that will have an important role to play against antibiotic-resistant and biofilm-producing bacteria.

\section{Dedication}

This article is dedicated to the loving memory of Dr Roderic MK Dale, former CEO and President, Oligos Etc. Inc. (Portland, OR, USA), who pioneered the design, and oversaw the early research and development of bisphophocins. Dr Dale was a visionary scientist whose love for science and passion about advancing drug discovery are a constant shining light to us all.

\section{Financial \& competing interests disclosure}

$S$ Parkinson and P Ditullio are CEO \& President and Vice President (Product Development) of Lakewood-Amedex, Inc., respectively, and therefore have financial involvement with the development and commercialization of bisphophocins. The authors have no other relevant affliations or financial involvement with any organization or entity with a financial interest in or financial conflict with the subject matter or materials discussed in the manuscript apart from those disclosed.

No writing assistance was utilized in the production of this manuscript.

\section{EXECUTIVE SUMMARY}

- Global crisis relating to the rapid rise in multiple drug-resistant bacteria necessitate the development of novel classes of antibiotics that have robust mechanisms of action, safety and efficacy profiles against antibiotic-resistant pathogens.

- Bisphosphocins are small, chemically stable nucleotide-based antibacterial agents that have broad-spectrum antibacterial activity mediated by bacterial cell membrane depolarization.

- Preclinical studies in animals suggest bisphosphocins are highly effective in the treatment of burn wound infections, suture wound infection and pulmonary infections caused by biofilm forming and antibiotic-resistant $P$. aeruginosa.

- Therapeutic efficacy of bisphosphocins was determined by survival rates and/or reduced bacterial loads in infected organs, tissues or blood.

- Bisphosphocins can be applied topically, systemically through intravenous administration, as well as by aerosol inhalation and suitable for treating both acute and chronic $P$. aeruginosa infections.

- When fully developed clinically, bisphosphocins will be a potent and broad-spectrum class of novel antimicrobials that will have an important role to play against antibiotic-resistant and biofilm-producing bacteria.

\section{References}

1 WHO. Antimicrobial resistance: no action today, no cure tomorrow. WHO Press, 7 April 2011.

http://www.who.int
2 Piddock LJ. The crisis of no new antibiotics - what is the way forward? Lancet. Infect. Dis. 12(3), 249-253 (2012).

3 Gilbert DN, Guidos RJ, Boucher HW et al. Infectious Diseases Society of America. The 10 x '20 initiative: Pursuing a global commitment to develop 10 new antibacterial drugs by 2020. Clin. Infect. Dis. 50(8), 1080-1083 (2010).

4 Mah T-FC, O'Toole GA. Mechanisms of biofilm resistance to antimicrobial agents. Trends Microbiol. 9(1), 34-39 (2001). 
5 Licking E. Getting a grip on bacterial slime. Business Week, 13 September, 98-100 (1999).

6 Perri MB, Dale R, Zervos MJ et al. In vitro activity of an investigation nucleic acid antibacterial compound $\mathrm{Nu}-2$ against multidrug resistant bacteria. Presented at: 39th Interscience Conference on Antimicrobial Agents and Chemotherapy. San Francisco, CA, USA, 1999 (abstract 1811).

7 Cao S, Sun L-Q, Wang M. Antimicrobial activity and mechanism of action of $\mathrm{Nu}-3$, a protonated modified nucleotide. Ann. Clin. Microbiol. Antimicrob. 10, 1 (2011).

8 Akiyoshi D, Dilo J, Di Tullio P. Novel bisphosphocin $\mathrm{Nu}-3$ demonstrates rapid killing of bacteria-encased in biofilm in vitro. Presented at: 53rd Interscience Conference on Antimicrobial Agents and Chemotherapy. Denver, CO, USA, 2013 (abstract F1701a).
9 Wurtz R, Karajovac E, Dacumos E et al. Nosocomial infections in a burn intensive unit. Burns 21, 180-184 (1995).

10 Dale RMK, Schnell G, Wong JP. Therapeutic efficacy of 'nubiotics' against burn wound infection by Pseudomonas aeruginosa. Antimicrob. Agents Chemother. 48(8), 2918-2923 (2004).

11 Moreau-Marquis S, Stanton BA, O’Toole GA. Pseudomonas aeruginosa biofilm formation in the cystic fibrosis airway. A short review. Pulm. Pharmacol. Ther. 21(4), 595-599 (2008).

12 Cash HA, Woods DE, McCoullough B et al. A rat model of chronic respiratory infection with Pseudomonas aeruginosa. Am. Rev. Respir. Dis. 119, 453-459 (1979).
13 Dale RMK, Schnell G, Zhang RJD et al. Therapeutic efficacy of aerosolized liposomeencapsulated nubiotic against pulmonary Pseudomonas aeruginosa infection. Therapy 4(4), 441-449 (2007).

14 Alipour M, Suntres ZE, Lafrenie RM et al. Attenuation of Pseudomonas aeruginosa virulence factors and biofilm by coencapsulation of bismuth-ethanedithiol with tobramycin in liposomes. J. Antimicrob. Chemother. 65(4), 684-693 (2010).

15 Beiras-Fernandez A, Vogt F, Sodian R et al. Daptomycin: a novel lipopeptide antibiotic against Gram-positive pathogens. Infect. Drug Resist. 3, 95-101 (2010).

16 Saravolatz LD, Stein GE, Johnson LB. Telavancin: a novel lipoglycopeptide. Clin. Infect. Dis. 49(12), 1908-1914 (2009). 